\title{
Automated Near-Field Scanning Algorithm for the EMC Analysis of Electronic Devices
}

\author{
Dirk Deschrijver Member, IEEE, Filip Vanhee Member, IEEE, Davy Pissoort Member, IEEE, Tom Dhaene Senior \\ Member, IEEE
}

\begin{abstract}
This paper presents an automated procedure to determine the electric or magnetic near field profile of electronic systems and devices in a given plane. It combines sequential sampling to determine the optimal coordinates of near field scan points at arbitrary coordinates in the scanning plane. The effectiveness of the approach is illustrated by applying it to both a simulated and measured printed circuit board example.
\end{abstract}

Index Terms-Electromagnetic compatibility, near field scanning, surrogate modeling, sequential sampling, Kriging

\section{INTRODUCTION}

A $\mathrm{S}$ modern electronic systems are combining more and more functionalities in an ever smaller area and at higher and higher frequencies, the risks for intra- and inter-system electromagnetic interference (EMI) issues keep on increasing. Therefore, obtaining an in-depth knowledge of the electromagnetic behavior of electronic components and (sub)systems very early in the design process is essential. Over the last decade, electromagnetic near-field (NF) scanning is gaining increasing interest for the assessment of the electromagnetic compatibility (EMC) behavior of electronic (sub)systems [1]. When compared to the classical EMC measurement techniques, NF scanning has a number of important advantages. First, there is no real need to perform the measurements in expensive (semi)-anechoic or reverberant chambers. Second, NF scanning allows to assess both the intra- and inter-system EMC behavior of the device under test. Third, equivalent radiation models can be derived from the NF patterns to predict the device's far-field radiation or for use as component models in commercial electromagnetic software tools. These models can comprise a set of magnetic and/or electric dipoles [2]-[9], a set of multi-poles [10], or a discretized set of electric and magnetic currents directly derived from the NF data based on the field-equivalence principle [11]-[14].

However, obtaining the requested, un-perturbed NF data in an efficient way is not straight-forward. Difficulties include the accurate measurement of both amplitude and phase of the NF components [12], and the overall time needed to scan a complete device with sufficient resolution to capture all relevant elements in the NF pattern. Also, field probes

Manuscript received April 2011; revised April 2011.

Dirk Deschrijver and Tom Dhaene are with the Department of Information Technology, Ghent University - IBBT, Sint Pietersnieuwstraat 41, 9000 Ghent, Belgium, email: \{dirk.deschrijver, tom.dhaene\}@intec.ugent.be. Filip Vanhee and Davy Pissoort are with the Flanders' Mechatronics Engineering Center at the Faculty of Engineering Technology of the University College KHBO, K.U. Leuven Association, email : davy.pissoort@khbo.be. This work was supported by the Fund for Scientific Research Flanders (FWO-Vlaanderen).

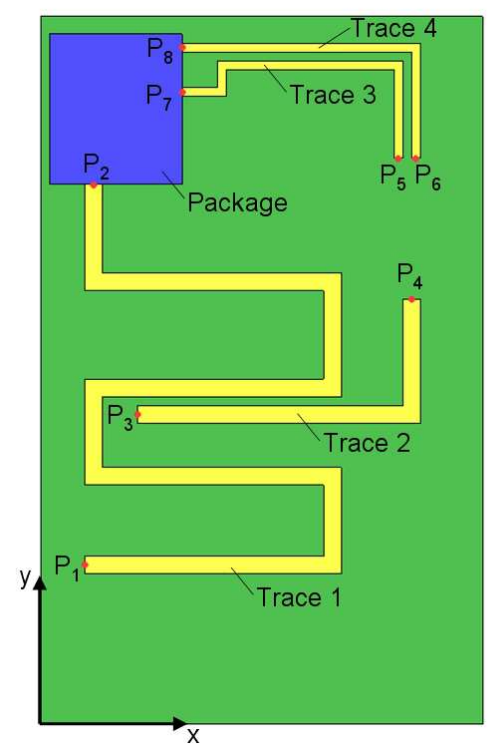

Fig. 1. Device under test : printed circuit board

themselves disturb the NFs and don't really measure one single field component directly [15]-[19], necessitating the use of calibration and compensation techniques.

In this paper, the problem of excessive measurement times for obtaining the 'raw' NF data (i.e. uncalibrated and/or un-compensated) is addressed. An automated sampling and modeling algorithm is proposed that (i) optimizes the NF scanning procedure without requiring any prior knowledge of the device under test, and (ii) allows to interpolate the raw NF data in arbitrary points in the scanning plane. These models can then be used as input for post-processing algorithms to compensate the influence of the NF probe, to derive amplitude and/or phase of other field components or to derive equivalent radiation models.

The proposed algorithm starts from a limited set of NF scan points and determines the optimal location of additional scan points in a sequential way. The advantage of using an advanced modeling strategy was already shown in [20] where artificial neural networks were used. A key advantage of the proposed algorithm is the sequential behavior. Indeed, for the conventionally used uniform sampling, it is not known a priori how many NF scan points are needed to characterize the device under test. Thanks to the sequential behavior, the proposed algorithm allows to evaluate at any moment the convergence of the obtained NF pattern and to implement an automatic stopping criterion for the NF scan. 


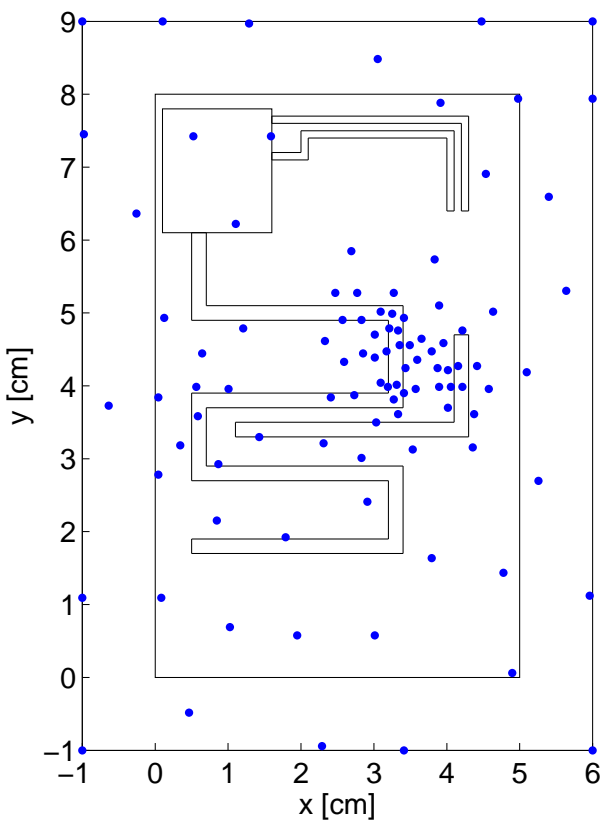

Fig. 2. Data sample distribution for $K=100$ samples

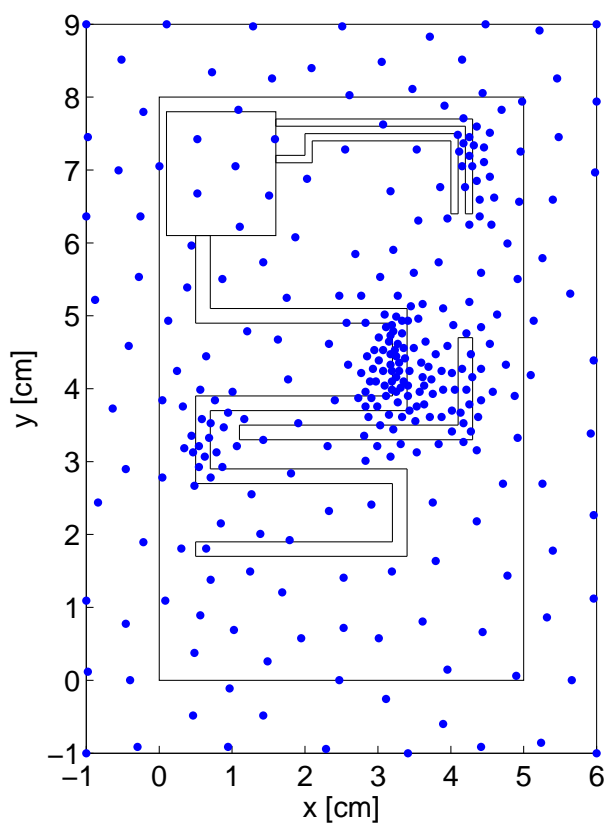

Fig. 3. Data sample distribution for $K=300$ samples

As additional NF scans points are selected, Kriging approximation models are calculated to model the sampled NF pattern. These models are fast to evaluate, and can be used to reconstruct the NF profile with a high-level resolution for arbitrary scan coordinates. Once these models have converged to a common solution, the selection of additional scan points is terminated and the final model is returned as a result.

To demonstrate the efficacy of the approach, the proposed NF scanning algorithm is applied to three different examples. Numerical results confirm that the method yields excellent sampling and modeling results. It is shown that the method

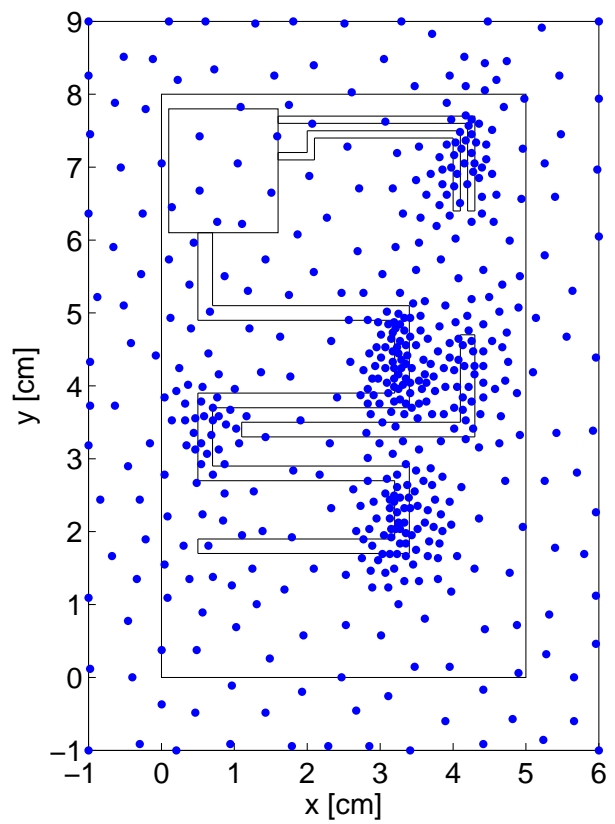

Fig. 4. Data sample distribution for $K=500$ samples

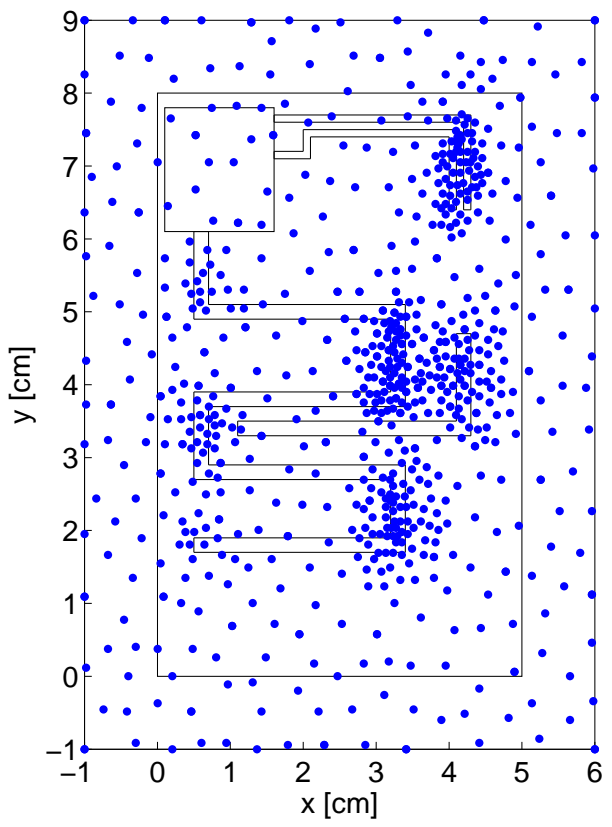

Fig. 5. Data sample distribution for $K=700$ samples

is applicable to both simulations and (noisy) measurements.

\section{Goal Statement And Preliminaries}

The goal of the sequential sampling algorithm, described in Sect. III, is to minimize the number of NF scan points, needed to characterize the overall electromagnetic behavior of the device under study. Each NF scan point, henceforth also called data sample, is represented as a tuple $(\boldsymbol{x},|F(\boldsymbol{x})|)$, where $\boldsymbol{x}$ is a vector that contains the horizontal, vertical, and height coordinates of the probe and $|F(\boldsymbol{x})|$ represents the amplitude 


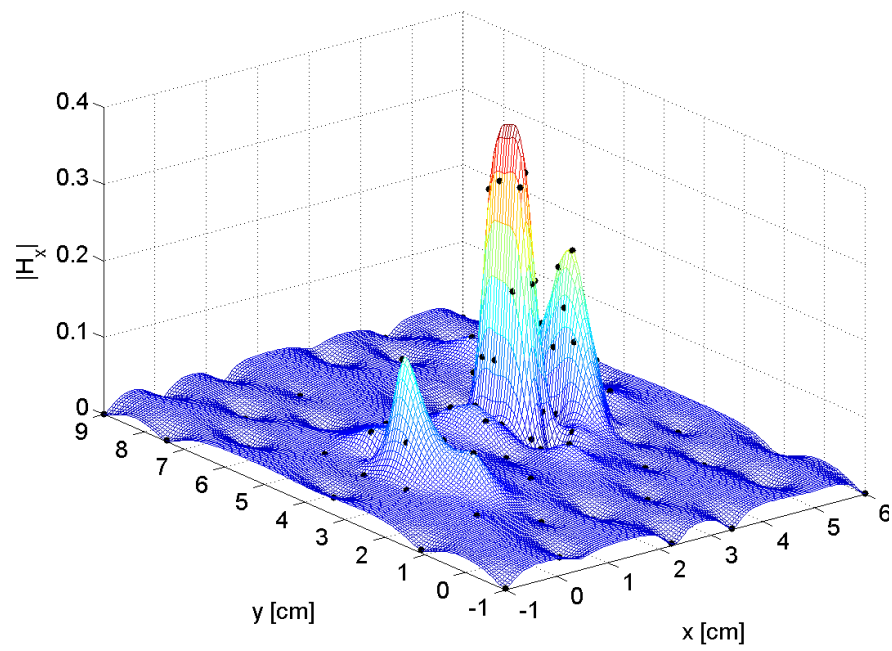

Fig. 6. Kriging approximation model, based on $K=100$ samples

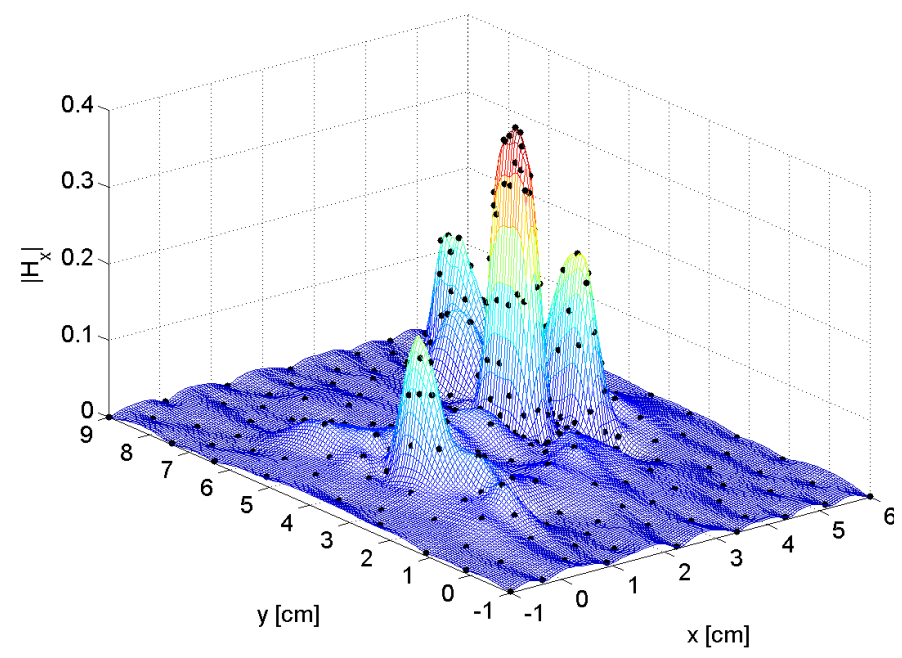

Fig. 7. Kriging approximation model, based on $K=300$ samples

of the electric $(E)$ or the magnetic $(H)$ field component. Each spatial coordinate in vector $\boldsymbol{x}$ is denoted by a superindex $x^{(n)}$.

Once a set of $K$ data samples $\left\{\left(\boldsymbol{x}_{k},\left|F\left(\boldsymbol{x}_{k}\right)\right|\right)\right\}_{k=1}^{K}$ is selected, the Kriging modeling algorithm presented in Sect. IV is applied to compute an accurate approximation model. This analytical model is fast to evaluate, and can be used to identify the near fields for arbitrary coordinates in the design space. Note that the term design space refers to the area of interest over which the coordinates $\boldsymbol{x}$ of the probe are varied.

\section{Sequential SAmpling Algorithm}

The sequential sampling algorithm starts by computing a small number of initial scan points according to a Latin hypercube design [21]. In successive steps, additional scan points are selected in a sequential way until the overall variation of the NF pattern is characterized. In order to sample the NF pattern as efficiently as possible, the robust sampling strategy from [22], [23] is applied to determine the optimal coordinates

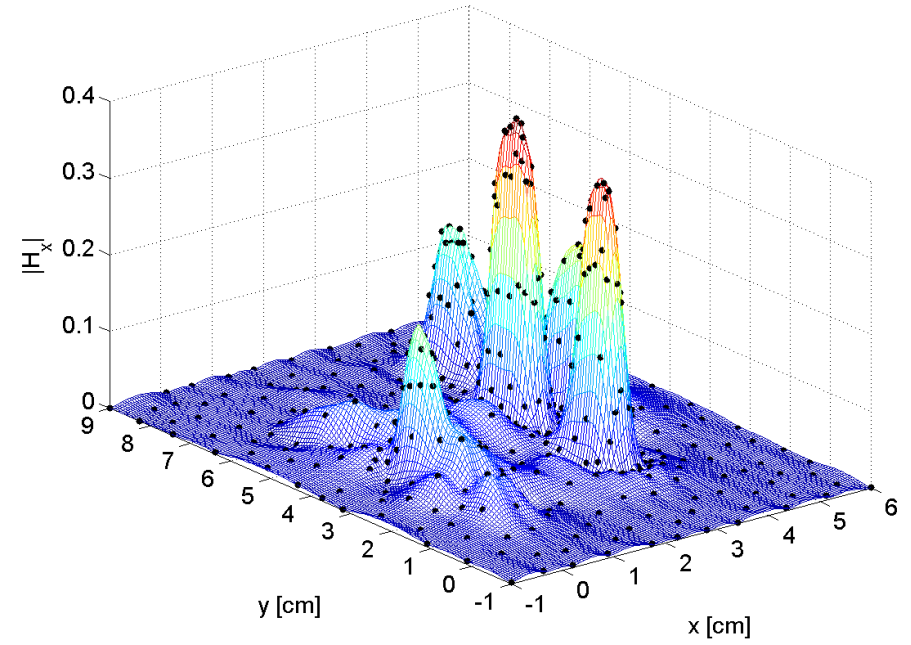

Fig. 8. Kriging approximation model, based on $K=500$ samples

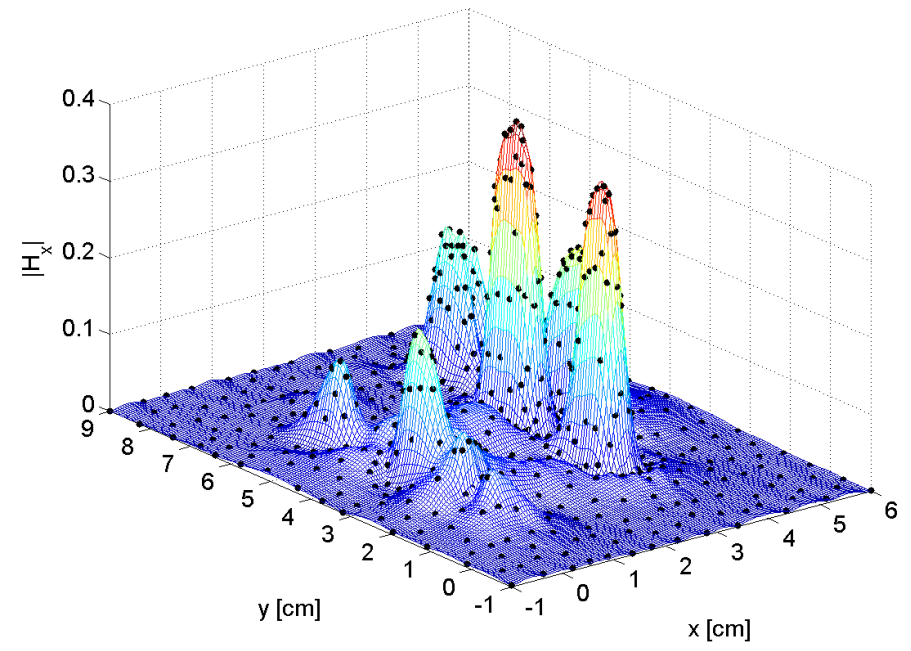

Fig. 9. Kriging approximation model, based on $K=700$ samples

of the scan points in a sequential way. It makes a balanced trade-off between exploration and exploitation criteria :

- Exploration is the act of exploring the design space in order to detect key regions that have not yet been identified before. It does not involve the actual pattern of the near-fields, but only the coordinates $\boldsymbol{x}$ of the scan points and their coverage of the design space. It ensures that all the scan points are spread as evenly as possible.

- Exploitation ensures that additional scans are performed in regions of the design space where the amplitude $|F(\boldsymbol{x})|$ is changing more rapidly. These regions often require a finer sampling density than regions with little variation.

For the exploration criterion, the density of data samples is quantified by computing a Voronoi tessellation of the data samples and by calculating the volume of each Voronoi cell. For the exploitation criterion, the dynamic variation of the near fields is quantified by computing simple local linear approximation models that are compared with the sampled NF pattern. Both criteria are combined into a unified metric 


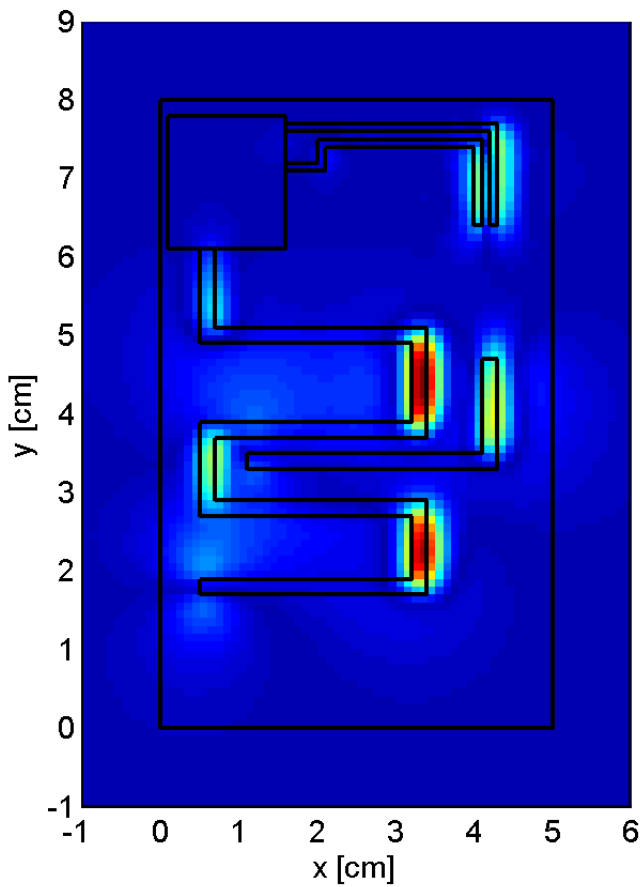

Fig. 10. NF pattern : uniform sampling of $\left|H_{x}\right|$ (7171 scan points)

that can be used to identify undersampled regions of the design space, and to determine the optimal location of additional scan points. Although a brief outline of this procedure is discussed below, the reader is referred to [23] for an in-depth discussion.

\section{A. Exploration - Voronoi Tessellations}

The density of data samples is assessed by computing a Voronoi tessellation [24] of the design space and by estimating the volume of each cell. Cells having a large volume correspond to regions in the design space that are sampled sparsely. Assume that a discrete and pairwise distinct set of coordinates $X=\left\{\boldsymbol{x}_{k}\right\}_{k=1}^{K}$ in the design space is given. Then the Voronoi cell $C_{k}$ of $\boldsymbol{x}_{k}$ contains all samples in the design space lying closer to $\boldsymbol{x}_{k}$ than any other sample in $X$. The complete set of cells $\left\{C_{k}\right\}_{k=1}^{K}$ tessellates the design space, and is called the Voronoi tessellation corresponding to $X$. To compute the volume of each Voronoi cell, the unbounded cells near the border of the parameter ranges are bounded. Then, the volume (Vol) of each cell is estimated by Monte Carlo methods [25].

To assess the density of the data samples around $\boldsymbol{x}_{k}$, the following normalized metric $V\left(\boldsymbol{x}_{k}\right) \in[0,1]$ is introduced

$$
V\left(\boldsymbol{x}_{k}\right)=\frac{\operatorname{Vol}\left(C_{k}\right)}{\operatorname{Vol}\left(C_{1}\right)+\ldots+\operatorname{Vol}\left(C_{K}\right)}
$$

Note that $V\left(\boldsymbol{x}_{k}\right)$ quantifies the portion of the design space that is contained within each Voronoi cell $C_{k}$ of coordinate $\boldsymbol{x}_{k}$.

\section{B. Exploitation - Local Linear Approximations}

Regions of the design space with a dynamic NF pattern are identified as follows. For each coordinate $\boldsymbol{x}_{k}$ in $X$, a suitable

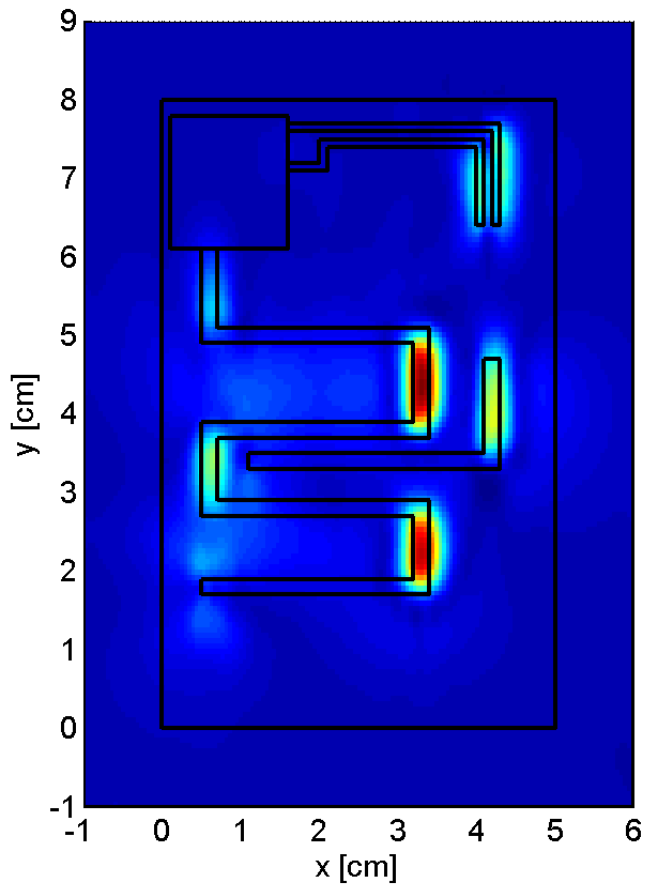

Fig. 11. NF pattern : sequential sampling of $\left|H_{x}\right|$ (700 scan points)

set of $V$ neighbouring coordinates $N\left(\boldsymbol{x}_{k}\right)$ is chosen.

$$
N\left(\boldsymbol{x}_{k}\right)=\left\{\boldsymbol{x}_{k v}\right\}_{v=1}^{V} \text { with } N\left(\boldsymbol{x}_{k}\right) \subset X \backslash\left\{\boldsymbol{x}_{k}\right\}
$$

These neighbours are chosen in such a way that each direction of the design space is covered equally well. Using these neighbours, the gradient $\nabla|F(\boldsymbol{x})|$ is estimated from data [26]

$$
\nabla|F(\boldsymbol{x})|=\left(\frac{\partial|F(\boldsymbol{x})|}{\partial x^{(1)}}, \frac{\partial|F(\boldsymbol{x})|}{\partial x^{(2)}}, \ldots, \frac{\partial|F(\boldsymbol{x})|}{\partial x^{(N)}}\right)
$$

and leads to the best local linear approximation $|\tilde{F}(\boldsymbol{x})|$ at $\boldsymbol{x}_{k}$

$$
|\tilde{F}(\boldsymbol{x})|=\left|F\left(\boldsymbol{x}_{k}\right)\right|+(\nabla|F(\boldsymbol{x})|)_{x_{k}}\left(\boldsymbol{x}-\boldsymbol{x}_{k}\right)
$$

Note that $(\nabla|F(\boldsymbol{x})|)_{x_{k}}=A^{-1} b$ is computed by fitting a hyperplane through coordinate $\boldsymbol{x}_{k}$, based on its $V$ neighbouring coordinates $\left\{\boldsymbol{x}_{k v}\right\}_{v=1}^{V}$, provided that $A(v, n)=\left(x_{k v}^{(n)}-x_{k}^{(n)}\right)$ and $b(v, 1)=\left|F\left(\boldsymbol{x}_{k v}\right)\right|$ for $v=1, . ., V$ and $n=1, \ldots, N$.

Once the gradient is estimated, the dynamical behavior around coordinate $\boldsymbol{x}_{k}$ is quantified by evaluating the local linear approximation in (4) and comparing the estimated NF pattern $|\tilde{F}(\boldsymbol{x})|$ with the sampled NF pattern $|F(\boldsymbol{x})|$ at the neighboring coordinates $\boldsymbol{x}_{k v}$. A large deviation metric

$$
\bar{W}\left(\boldsymbol{x}_{k}\right)=\sum_{v=1}^{V}|| \tilde{F}\left(\boldsymbol{x}_{k v}\right)|-| F\left(\boldsymbol{x}_{k v}\right)||
$$

indicates regions where the data is varying more rapidly. To obtain a normalized metric $W\left(\boldsymbol{x}_{k}\right) \in[0,1]$, one defines

$$
W\left(\boldsymbol{x}_{k}\right)=\frac{\bar{W}\left(\boldsymbol{x}_{k}\right)}{\bar{W}\left(\boldsymbol{x}_{1}\right)+\ldots+\bar{W}\left(\boldsymbol{x}_{K}\right)}
$$

This metric $W\left(\boldsymbol{x}_{k}\right)$ quantifies the portion of the dynamic variation in the response that is located near coordinate $\boldsymbol{x}_{k}$. 


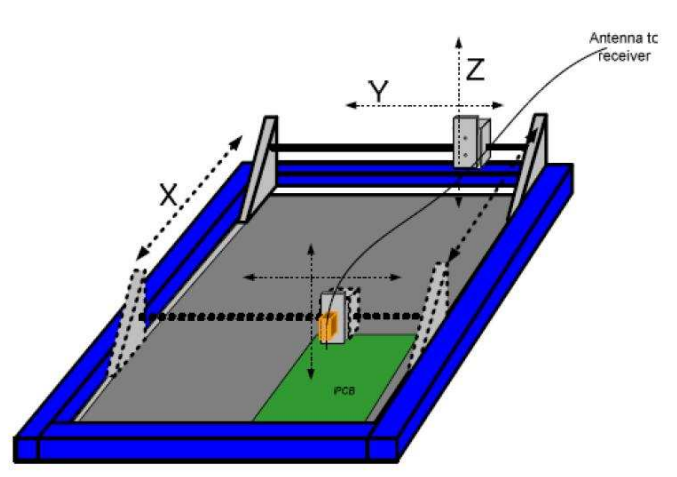

Fig. 12. NF scanning system

\section{Sequential Data Sample Selection}

The exploration-based metric $V(\boldsymbol{x})$ in (1) quantifies data samples according to the size of their corresponding Voronoi cell, while the exploitation-based metric $W(\boldsymbol{x})$ in (6) quantifies samples according to the local variation of the NF pattern. Both are combined into a global metric that is used for ranking.

$$
G(\boldsymbol{x})=V(\boldsymbol{x})+W(\boldsymbol{x})
$$

Data samples associated with large values of (7) are located in regions which are likely undersampled, whereas the smaller values of (7) correspond to regions that are sampled sufficiently dense. If coordinate $\boldsymbol{x}_{m} \in X$ corresponds to the maximum value of (7), then the algorithm select an additional data sample inside the Voronoi cell $C_{m}$. Its exact coordinate is chosen in such a way that the distance from the neighbours $N\left(\boldsymbol{x}_{m}\right)$ is maximized. This coordinate is then propagated to the NF scanner, which collects the amplitude of the near field and adds a new tuple to the set of selected data samples. This process of adding data samples is repeated iteratively.

\section{Kriging Modeling Algorithm}

Once a set of data samples is obtained from the sequential sampling algorithm, an analytic approximation model can be computed by Kriging. Kriging, also known as Gaussian Process regression, is a geostatistical modeling technique that originates from geology and mining [27]. Later on, it has been successfully adopted in several other application areas, including electrical engineering. This paper shows that the technique is also well suited for the modeling of sampled nearfield patterns. Given a sparse set of $K$ selected data samples, a Kriging model $M(\boldsymbol{x})$ of the following form is computed

$$
M(\boldsymbol{x})=\sum_{p=1}^{P} \beta_{p} f_{p}(\boldsymbol{x})+\sum_{k=1}^{K} \alpha_{k} \phi\left(\theta, \boldsymbol{x}_{k}, \boldsymbol{x}\right)
$$

where the coefficients $\beta_{p}$ and $\alpha_{k}$ are estimated from the data by a generalized least squares procedure. The first part of (8) corresponds to a linear regression with respect to the basis functions $f_{p}(\boldsymbol{x})$. This is typically chosen to be a low-order polynomial or a constant. The second part of (8) can be seen as a localized deviation from the regression component, and is formulated as a summation of $K$ shifted instances of

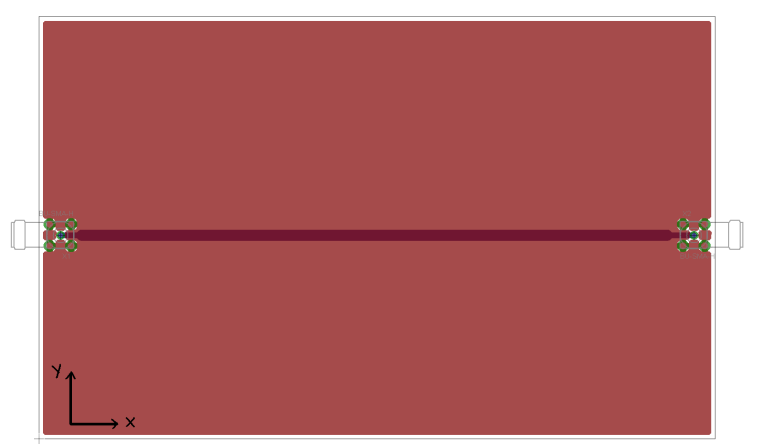

Fig. 13. Device under test : straight microstrip

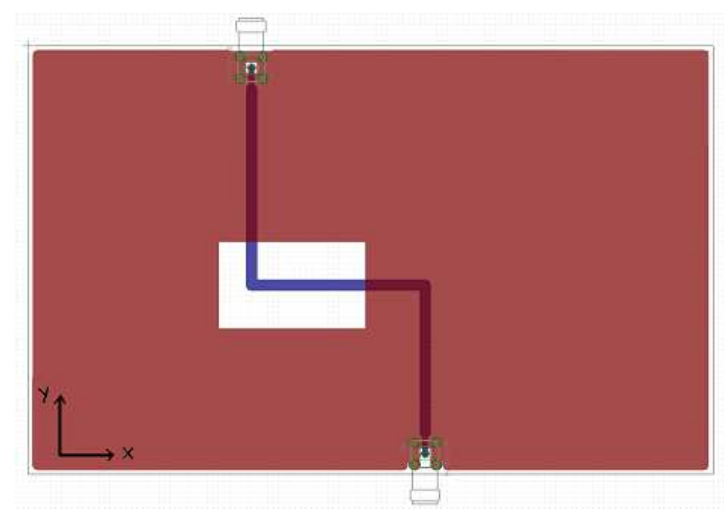

Fig. 14. Device under test : bended microstrip over slot

the correlation function, each centered at one data sample. Considering the nature of the near-field patterns, it seems natural to choose the correlation function as a Gaussian

$$
\phi\left(\theta, \boldsymbol{x}_{k}, \boldsymbol{x}\right)=\prod_{n=1}^{N} \mathrm{e}^{-\theta_{n}\left|x_{k}^{(n)}-x^{(n)}\right|^{2}}
$$

where $n$ represents an index that is used to iterate all coordinates of the probe, stored in vector $\boldsymbol{x}$. Note that the parameters $\theta_{n}$ are identified by maximum likelihood estimation [28].

\section{EXAMPle : PRINTED Circuit BoARD}

As a first example, the algorithm is applied to assess the electromagnetic behavior of the printed circuit board (PCB), shown in Fig. 1. The PCB comprises a FR4 substrate (relative permittivity $=4.2$ ) of $8 \mathrm{~cm}$ by $5 \mathrm{~cm}$ and with a thickness of $1.5 \mathrm{~mm}$. The bottom layer is a ground plane. On the top layer there are 4 traces of various widths, shapes and lengths, which are terminated by different impedances. Traces 1 and 2 have a width of $2 \mathrm{~mm}$, while traces 3 and 4 have a width of $1 \mathrm{~mm}$. Ports $P_{1}$ and $P_{4}$ are $1 \mathrm{~V}$ voltage sources with an internal impedance of $50 \Omega$. Ports $P_{5}$ and $P_{6}$ are $1 \mathrm{~V}$ voltage sources with an internal impedance of $100 \Omega$. Port $P_{3}$ is a 50 $\Omega$ resistor, port $P_{2}$ is a $360 \Omega$ resistor, while both ports $P_{7}$ and $P_{8}$ are shorts. Finally, the PCB comprises in the top left corner a rectangular dielectric block of $15 \mathrm{~mm}$ by $17 \mathrm{~mm}$ and a height of $1 \mathrm{~mm}$, representing e.g. a package. Based on an FDTD simulation with Agilent's EMpro [29], the amplitudes of all field components $\left|F_{x}\right|$ are gathered at $2 \mathrm{~mm}$ above the 
TABLE I

OVERVIEW OF SCANNING RESULTS

\begin{tabular}{|c||c|c|}
\hline Step & Scan points & Meas. time \\
\hline $1.0 \mathrm{~mm}$ & 13741 & 1 hour \\
\hline $1.5 \mathrm{~mm}$ & 6161 & $26.4 \mathrm{~min}$ \\
\hline $2.0 \mathrm{~mm}$ & 3496 & $15.0 \mathrm{~min}$ \\
\hline $2.5 \mathrm{~mm}$ & 2257 & $9.6 \mathrm{~min}$ \\
\hline $3.0 \mathrm{~mm}$ & 1581 & $6.8 \mathrm{~min}$ \\
\hline
\end{tabular}

PCB over an area of $10 \mathrm{~cm}$ by $7 \mathrm{~cm}$, and this at a frequency of $900 \mathrm{MHz}$. For convenience of presentation, the height of the NF probes is fixed such that the vector $\boldsymbol{x}$ is two-dimensional. In the illustrations discussed below, only the amplitude of $\left|H_{x}\right|$ is considered, however the same procedure can be applied to model any component of the NF pattern.

\section{A. Sampling and Modeling}

As mentioned in Sect. III, the sequential sampling algorithm starts by gathering a limited set of 24 initial data samples (i.e. NF scan points). These initial samples include the 4 corners points of the design space, as well as 20 additional samples that are chosen according to an optimized Latin Hypercube design [30]. In a sequential way, additional samples are selected until the overall behavior of the NF pattern is well characterized. It is seen from comparing Figs. 2-5 with Figs. 6-9 that the selected data samples are distributed more densely in the regions where the NF pattern is changing more rapidly. Nevertheless, the algorithm ensures that a good sampling coverage of the overall design space is preserved.

Each time a new batch of additional data samples is selected, a Kriging approximation model is calculated as proposed in Sect. IV. It is observed from Figs. 6-9 that the Kriging model provides an excellent approximation of the data, and captures all the variation in the NF pattern in an accurate way. As expected, the model becomes a better approximation of the true NF pattern as the number of selected data samples grows. At the same time, the error drops until it eventually becomes small enough to conclude that the NF pattern is accurately captured. Once the Kriging models have converged to a common solution, it is assumed that all changes in the $\mathrm{NF}$ pattern are detected and the algorithm terminates. Note that any kind of predefined error measure (e.g. relative error, RMS , ...) can be used to define a suitable stopping criterion.

Based on the final Kriging model, it is possible to show a high-resolution cartography of the design space. Such figures visualize the strength of the magnetic field and can be used to study shielding and coupling phenomena, as well as the localization of radiation sources and hotspots. As an example, Fig. 11 (basically a top-view of Fig. 9) shows the carthography of the final model after selecting 700 data samples. As a validation test, the NF pattern when using a classical uniform sampling of 7171 data samples is shown in Fig. 10. The good agreement confirms that the algorithm is indeed able to accurately capture the NF pattern by performing fewer scans.

\section{EXAMPLE : MiCROSTRIP LINES}

As a second example, the proposed method is applied to a measurement case. Fig. 12 shows the NF scanning system that was used in this paper. It consists of a $\mathrm{CNC}$ milling machine that was rebuilt into a NF scanning system. To do this, the miller and its suspension were removed and replaced by a head to which a NF probe can be attached. The head can be moved automatically in three dimensions above the device under test to perform the measurements. The magnetic NF probe from Langer EMV-Technik (RF-U 2.5-2) has a resolution of about $0.5 \mathrm{~mm}$ in the frequency range of $30 \mathrm{MHz}$ up to $3 \mathrm{GHz}$. The probe is connected to a Rohde \& Schwarz EMI receiver. Only the amplitude of the tangential magnetic fields is measured at a height of $2 \mathrm{~mm}$ above the PCB under test. All the measurements are done at a frequency of $200 \mathrm{MHz}$.

The algorithm is applied to a set of simple PCBs, shown in Figs. 13 and 14. Both of them are comprised of $50 \Omega$ microstrips on a $15 \mathrm{~cm}$ by $9 \mathrm{~cm}$ FR 4 substrate of $1.5 \mathrm{~mm}$ thickness. To create sufficient radiation, some basic EMC rules were violated on the test PCBs (like e.g. routing the microstrip over a slot in the ground plane). The first PCB is a simple straight microstrip line. The second PCB is a bended microstrip, which is routed over a slot in the ground plane.

\section{A. Sampling and Modeling}

This case concerns modeling the amplitudes of the tangential magnetic fields $\left(\left|H_{x}\right|\right.$ and $\left.\left|H_{y}\right|\right)$ of the straight microstrip line (Fig. 13) and the bended microstrip line (Fig. 14). The measurements are performed at a height of $2 \mathrm{~mm}$ above the PCB under test, using a $75 \times 45$ uniform sampling in the horizontal $(x)$ and vertical $(y)$ direction of the probe. This corresponds to a scanning resolution of approximately $2 \mathrm{~mm}$ in each direction, and gives a total amount of 3375 scan points per field component. A cartography of the resulting NF patterns is shown in Figs. 15-16 and Figs. 17-18 respectively.

In order to demonstrate the advantages of the new approach, the sequential algorithm is applied to sample and to model the noisy NF patterns in a fully automated way. The algorithm terminates after measuring only 350 scan points, and a high resolution carthography of the NF pattern is reconstructed in Figs. 19-20 and Figs. 21-22 respectively. It is interesting to note that the algorithm preferable chooses scan points in the regions where the NF pattern is changing more rapidly, which are not necessarily the regions with the strongest fields. Even though fewer NF scans are performed, a very good agreement with the uniformly sampled field patterns can be observed.

\section{B. Timing results}

Table I shows an overview for the number of performed NF scans, in the case where the PCB is scanned on a uniform grid with a measurement step that is varied inbetween $1 \mathrm{~mm}$ and $3 \mathrm{~mm}$. It is found that the actual number of data samples (and the time needed to measure these points) is highly dependent on this step size. Unfortunately, the optimal value of the step is not known in advance. Thanks to its sequential nature, the proposed algorithm overcomes this problem. For the PCB under study, the algorithm showed that the NF pattern was already converged after 350 scan points. In order to obtain a comparable number of samples with uniform sampling, the step size must be increased significantly. However, if the step 


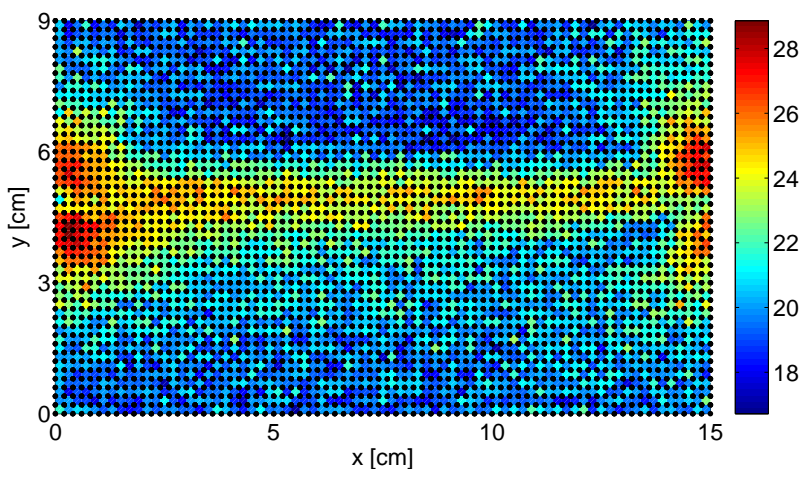

Fig. 15. Straight microstrip : uniform sampling of $\left|H_{x}\right|$ (3375 scan points)

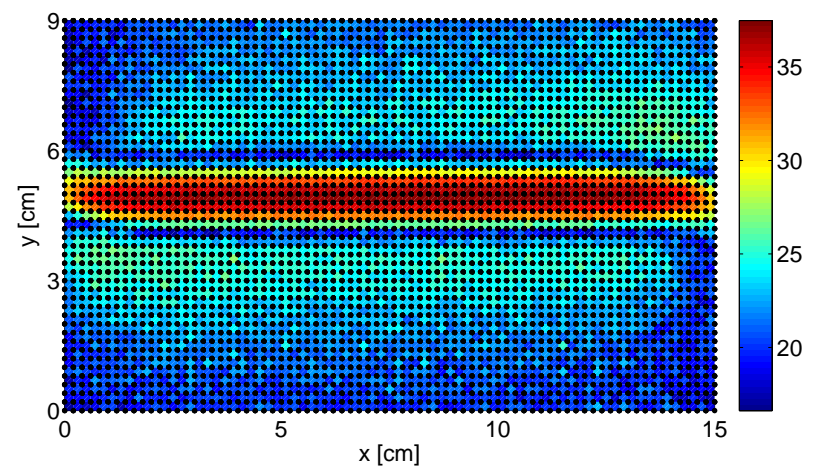

Fig. 16. Straight microstrip : uniform sampling of $\left|H_{y}\right|$ (3375 scan points)

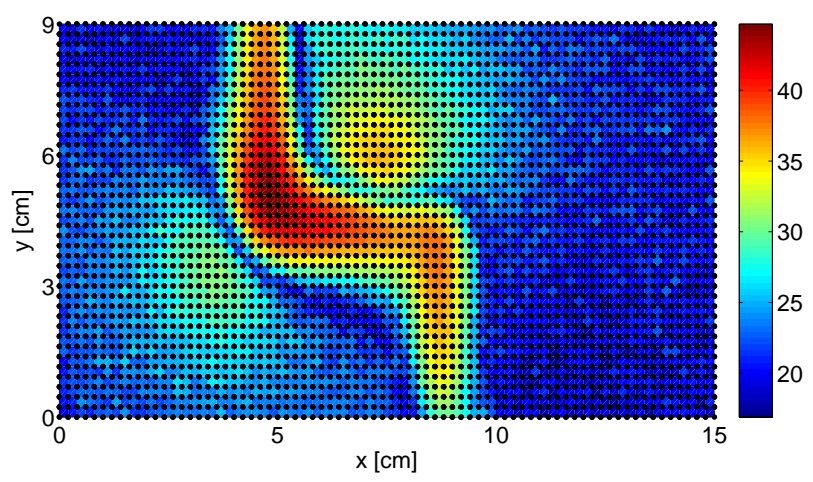

Fig. 17. Bended microstrip : uniform sampling of $\left|H_{x}\right|$ (3375 scan points)

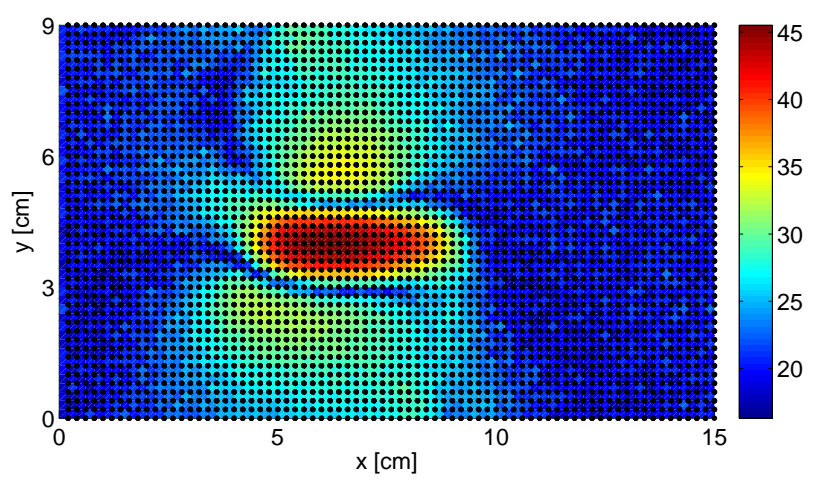

Fig. 18. Bended microstrip : uniform sampling of $\left|H_{y}\right|$ (3375 scan points)

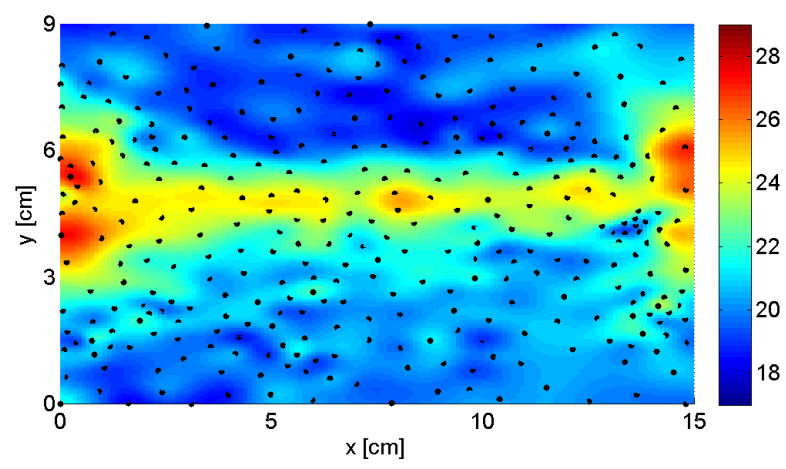

Fig. 19. Straight microstrip : sequential sampling of $\left|H_{x}\right|$ (350 scan points)

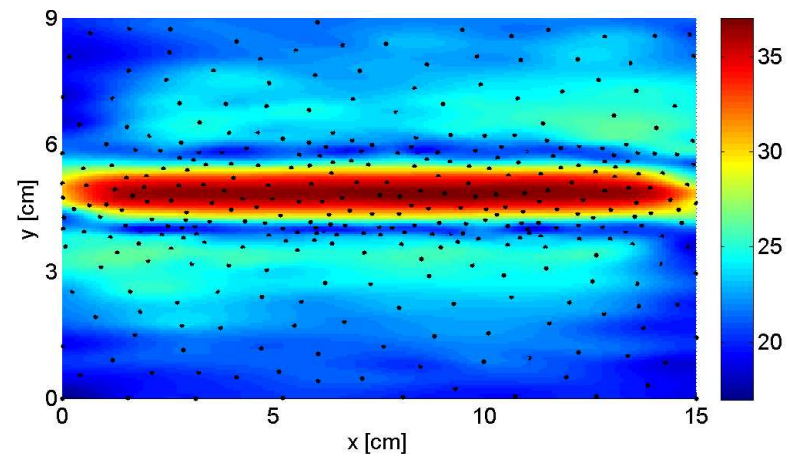

Fig. 20. Bended microstrip : sequential sampling of $\left|H_{y}\right|$ (350 scan points)

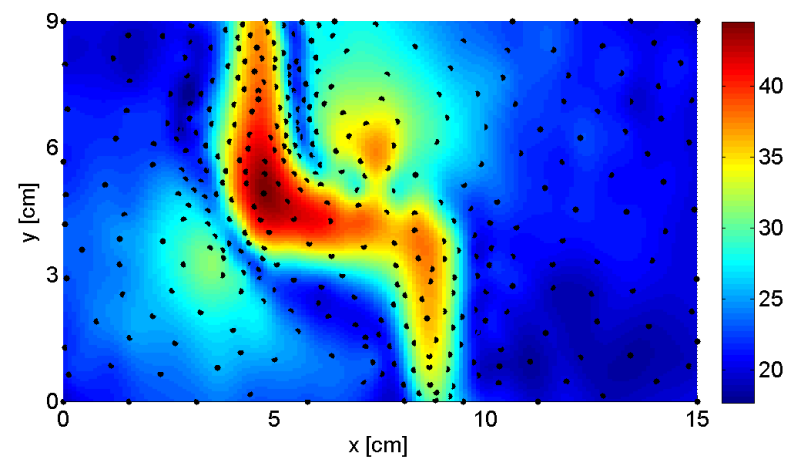

Fig. 21. Bended microstrip : sequential sampling of $\left|H_{x}\right|$ (350 scan points)

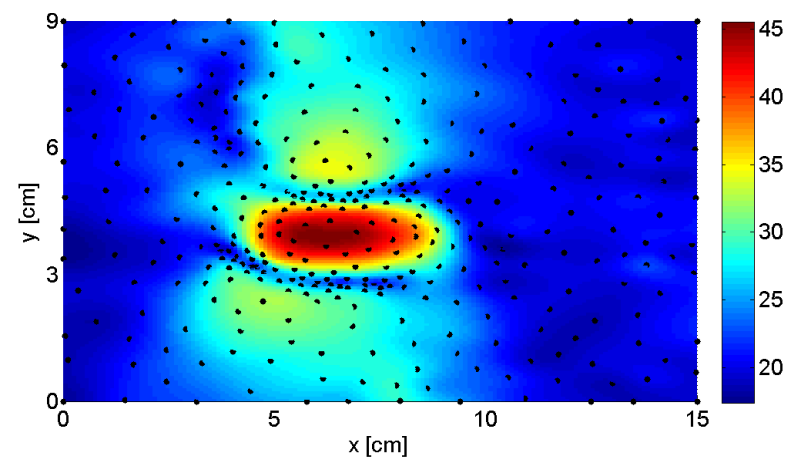

Fig. 22. Bended microstrip : sequential sampling of $\left|H_{y}\right|$ (350 scan points) 
size is chosen too large, the associated Kriging model will be less accurate than the sequentially built model, due to local undersampling of the NF pattern. To avoid such inaccuracies, the automated algorithm presented in this paper is preferable.

A possible drawback of the sequential algorithm is that some extra time is lost due to the extra time needed to move the NF probe between arbitrary positions that are sometimes far away from each other. Hence, a possible improvement for future work could be to optimize the sequence of measurements with respect to the movement of the NF probe.

\section{IMPLEMENTATION AND DISCUSSION}

An open source MATLAB implementation of the modeling techniques in Sect. III and IV is made publicly available for non-commercial, personal, academic use (AGPLv3 license) [31]. It can be downloaded as "SUMO Toolbox"from [32].

\section{CONCLUSIONS}

An automated near-field scanning procedure for the EMC analysis of electronic devices is presented. The method applies a sequential sampling strategy, in order to minimize the number of NF scan points needed to characterize the electromagnetic behaviour of the device under test. Based on the selected scans points, the Kriging modeling algorithm is applied to reconstruct the near field pattern with an improved resolution. The effectiveness and benefits of the new approach are demonstrated by applying it to a set of PCB examples.

\section{REFERENCES}

[1] D. Baudry, C. Archambal, A. Louis, B. Mazari and P. Eudeline, "Applications of the near-field techniques in EMC investigations", IEEE Transactions on Electromagnetic Compatibility, vol. 49, no. 3, pp. 485493, Aug 2006.

[2] Y. Vives-Gilabert, C. Archambal, A. Louis, F. de Daran, P. Eudeline and B. Mazari, "Modeling Magnetic Radiations of Electronic Circuits using Near-Field Scanning Method", IEEE Transactions on Electromagnetic Compatibility, vol. 49, no. 2, pp. 391-400, Nov 2007.

[3] H. Weng, D. G. Beetner, R. E. DuBroff and J. Shi, "Estimation of High-Frequency Currents From Near-Field Scan Measurements", IEEE Transactions on Electromagnetic Compatibility, vol. 49, no. 4, pp. 805815, Nov 2007.

[4] Y. Vives-Gilabert, C. Archambal, A. Louis, P. Eudeline and B. Mazari, "Modeling Magnetic Emissions Combining Image Processing and an Optimization Algorithm", IEEE Transactions on Electromagnetic Compatibility, vol. 51, no. 4, pp. 909-918, Nov 2009.

[5] I. Scriven, J. Lu and A. Lewis, "Electromagnetic Noise Source Approximation for Finite-Difference Time-Domain Modeling Using NearField Scanning and Particle Swarm Optimization”, IEEE Transactions on Electromagnetic Compatibility, vol. 52, no. 1, pp. 89-97, Feb 2010.

[6] X. Tong, D. W. P. Thomas, A. Nothofer, P. Sewell and C. Christopoulos, textquotedblleft Modeling Electromagnetic Emissions From Printed Circuit Boards in Closed Environments Using Equivalent Dipoles ", IEEE Transactions on Electromagnetic Compatibility, vol. 52, no. 2, pp. 462470, May 2010

[7] A. Ramanujan, Z. Riah, A.Louis and B. Mazari, "Modeling the Electromagnetic Radiation of Passive Microwave Components Using a Near-Field Scanning Method ", IEEE Transactions on Electromagnetic Compatibility, vol. 52, no. 4, pp. 1056-1059, Nov. 2010

[8] P.F. Lopez, C. Archambal, D. Baudry, S. Verdeyme and B. Mazari, "Simple Electromagnetic Modeling Procedure: From Near-Field Measurements to Commercial Electromagnetic Simulation Tool", IEEE Transactions on Instrumentation and Measurements, vol. 59, no. 12, pp. 3111-3121, Dec 2010.

[9] A. Ramanujan, Z. Riah, A.Louis and B. Mazari, "Computational Optimizations Towards an Accurate and Rapid Electromagnetic Emission Modeling", Progress in Electromagnetics Research B, vol. 27, 365-384, 2011.
[10] J.R. Regué, M. Ribó, J.M. Garell and A. Martin, "A Genetic Algorithm Based Method for Source Identification and Far-Field Radiation Emissions Prediction From Near-Field Measurements for PCB Characterization ", IEEE Transactions on Electromagnetic Compatibility, vol. 43, no. 4, pp. 520-530, Nov 2001.

[11] P.A. Barrière, J.J. Laurin and Y. Goussard, "Mapping of Equivalent Currents on High-Speed Digital Printed Circuit Boards Based on Near-Field Measurements ", IEEE Transactions on Electromagnetic Compatibility, vol. 51, no. 3, pp. 649-658, Aug 2009.

[12] Y. Alvarez, F. Las-Heras and M.R. Pino, "The Source Reconstruction Method for Amplitude-Only Field Measurements ", IEEE Transactions on Antennas and Propagation, vol. 58, no. 8, pp. 2776 - 2781, Aug 2010.

[13] Y. Alvarez, M. Rodríguez, F. Las-Heras and M.H. Fernando, "On the Use of Source Reconstruction Method for Estimating Radiated EMI in Electronic Circuits ", IEEE Transactions on Instrumentation and Measurement, vol. 59, no. 12, pp. 3174 - 3183, Dec 2010.

[14] H. Weng, D.G. Beetner and R.E. DuBroff, "Prediction of Radiated Emissions Using Near-Field Measurements ", IEEE Transactions on Electromagnetic Compatibility (IEEE Early Access), 2011.

[15] J. Shi, M.A. Craft, K.P. Slattery, M. Yamaguchi and R.E. DuBroff, "Calibration and Compensation of Near-Field Scan Measurements", IEEE Transactions on Electromagnetic Compatibility, vol. 47, no. 3, pp. 642-650, Aug 2005.

[16] D. Baudry, A. Louis and B. Mazari, "Characterization of the OpenEnded Coaxial Probe Used for Near-Field Measurements in EMC Applications ", Progress in Electromagnetics Research, PIER 60, 311333,2006

[17] A. Tankielun, H. Garbe and W. John, "Calibration of Electric Probes for Post-Processing of Near-Field Scanning Data ", Proceedings of the 2006 International Symposium on EMC, pp. 119-124, 2006.

[18] S. Jarrix, T. Dubois, R. Adam, P. Nouvel, B. Azaïs and D. Gasquet, "Probe Characterization for Electromagnetic Near-Field Studies ", IEEE Transactions on Instrumentation and Measurement, vol. 59, no. 2, pp. 292-300, Feb 2010

[19] H. Weng, D.G. Beetner, and R.E. DuBroff, "Frequency-Domain Probe Characterization and Compensation Using Reciprocity ", IEEE Transactions on Electromagnetic Compatibility, vol. 53, no. 1, pp. 2-10, Feb 2011.

[20] R. Brahimi, A. Kornaga, M. Bensetti, D. Baudry, Z. Riah, A. Louis and B. Mazari, "Postprocessing of Near-Field Measurement Based on Neural Networks", IEEE Transactions on Instrumentation and Measurement, vol. 60, no. 2, pp. 539-546, Feb 2011.

[21] A. Forrester, A. Sobester and A. Keane, "Engineering Design via Surrogate Modelling : A Practical Guide", Wiley, 2008.

[22] K. Crombecq, D. Gorissen, L. De Tommasi and T. Dhaene, "A Novel Sequential Design Strategy for Global Surrogate Modeling", Proceedings 41th Conference on Winter Simulation, pp. 731-742, 2009.

[23] D. Deschrijver, K. Crombecq, H. M. Nguyen, T. Dhaene, "Adaptive Sampling Algorithm for Macromodeling of Parameterized S-Parameter Responses", IEEE Transactions on Microwave Theory and Techniques, vol. 59, no. 1, pp. 39-45, Jan 2011.

[24] F. Aurenhammer, "Voronoi Diagrams - A Survey of a Fundamental Geometric Data Structure", ACM Computing Surveys, vol. 23, no. 3, pp. 345-405, 1991.

[25] N. Metropolis and S. Ulam, "The Monte Carlo Method", Journal of American Statistical Association, vol. 44, no. 247, pp. 335-341, 1949.

[26] T. Hachisuka, W. Jarosz, R. P. Weistroffer, K. Dale, G. Humphreys, M. Zwicker, H. W. Jensen, "Multidimensional Adaptive Sampling and Reconstruction for Ray Tracing", ACM Transactions on Graphics, vol. 27, no. 3, 10 pages, 2008.

[27] M. A. Oliver and R. Webster, "Kriging: a method of interpolation for geographical information system", International Journal of Geographical Information Systems, vol. 4, no. 3, pp. 313-332, 1990.

[28] I. Couckuyt, F. Declercq, T. Dhaene, H. Rogier, L. Knockaert, "Surrogate-based Infill Optimization applied to Electromagnetic Problems", International Journal of $R F$ and Microwave Computer-Aided Engineering, vol. 20, no. 5, pp. 492-501, Sep 2010.

[29] Agilent EEsof EDA, Electromagnetic Professional (EMPro) Software, Agilent Technologies Inc, Santa Rosa, CA (USA).

[30] K. Crombecq, T. Dhaene, "Generating Sequential Space-Filling Designs using Genetic Algorithms and Monte Carlo Methods", Proceedings Simulated Evolution and Learning conference, pp. 80-84, 2010.

[31] D. Gorissen, K. Crombecq, I. Couckuyt, T. Dhaene and P. Demeester, "A Surrogate Modeling and Adaptive Sampling Toolbox for Computer Based Design", Journal of Machine Learning, vol. 11, pp. 2051-2055, Jul 2010. 
[32] Surrogate Modeling Toolbox v. 7.0, http://sumo.intec.ugent.be, Ghent University - IBBT, Ghent (Belgium), Mar 2011.

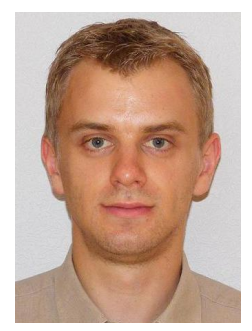

Dirk Deschrijver was born in Tielt, Belgium, on September 26, 1981. He received the Master degree (licentiaat) and $\mathrm{PhD}$ degree in Computer Science in 2003 and 2007 respectively, from the University of Antwerp in Antwerp, Belgium. During the period from May-October 2005, he was as a Marie Curie Fellow in the Scientific Computing group at the Eindhoven University of Technology in Eindhoven, The Netherlands. He is now working as an FWO post-doctoral research fellow in the Department of Information Technology (INTEC) at Ghent University in Belgium. His research interests include robust parametric macromodeling, rational least-squares approximation, orthonormal rational functions, system identification and broadband macromodeling techniques.

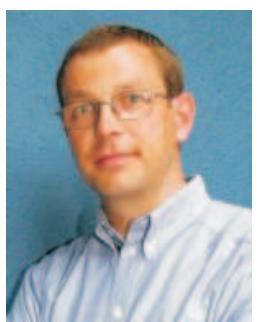

Tom Dhaene (M'94, SM'05) was born in Deinze, Belgium, on June 25, 1966. He received the Ph.D. degree in electrotechnical engineering from the University of Ghent, Ghent, Belgium, in 1993. From 1989 to 1993, he was Research Assistant at the University of Ghent, in the Department of Information Technology, where his research focused on different aspects of full-wave electro-magnetic circuit modeling, transient simulation, and time-domain characterization of high-frequency and high-speed interconnections. In 1993, he joined the EDA company Alphabit (now part of Agilent). He was one of the key developers of the planar EM simulator ADS Momentum. Since September 2000, he has been a Professor in the Department of Mathematics and Computer Science at the University of Antwerp, Antwerp, Belgium. Since October 2007, he is a Full Professor in the Department of Information Technology (INTEC) at Ghent University, Ghent, Belgium. As author or co-author, he has contributed to more than 220 peer-reviewed papers and abstracts in international conference proceedings, journals and books.

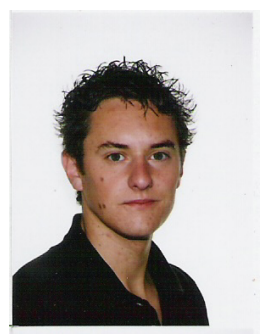

Filip Vanhee (M '11) was born in Belgium in 1983. $\mathrm{He}$ received the M.S. degree in electronics from the University College KHBO (Ostend, Belgium) in 2005. He is currently working towards the Ph.D. degree in electronic engineering from the K.U. Leuven, Leuven, Belgium. Since 2005, he is research assistant at the Flanders' Mechatronics Engineering Center at the KHBO, which is a laboratory focusing on global reliability of electronic systems. His main research interests include electromagnetic compatibility and environmental testing.

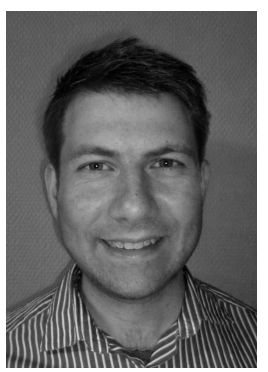

Davy Pissoort (M'09) was born in Dendermonde, Belgium on June 12th 1978. He received the M.S and $\mathrm{Ph} . \mathrm{D}$. degrees in electrical engineering from Ghent University, Ghent, Belgium, in 2001 and 2005, respectively. From October 2005 to October 2006, he was a Postdoctoral Researcher with the Fund for Scientific Research-Flanders (Belgium) (FWO-Vlaanderen) at the Department of Information Technology, Ghent University. In November 2006, he joined the EEsof-EDA Department, Agilent Technologies, Ghent, Belgium, as a Research Engineer. Since August 2009, he is with the KHBO, Belgium where he is head of the Flanders' Mechatronics Engineering Center. Since September 2009, he is also affiliated researcher at the ESAT department of the Katholieke Universiteit Leuven. His current research interests include the development of fast and efficiënt electromagnetic simulation methods, electromagnetic compatibility, signal and power integrity, as well as the analysis and testing of the mechanical and thermal reliability of electronic modules. 